\title{
Influence of reproductive tract obstruction on expression of epididymal proteins and their restoration after patency
}

\begin{abstract}
Bing-Kun Li ${ }^{1}$, Xiang Wang ${ }^{2}$, Chun-Xiao Liu ${ }^{1}$, Shao-Bo Zheng ${ }^{1}$, Hu-Lin Li ${ }^{1}$, Li-Ping Li $^{2}$ and A-Bai Xu ${ }^{1}$
Vasectomy is a simple and reliable method of male contraception. A growing number of men after vasectomy request vasectomy reversal due to various reasons. The pregnancy rate is lower than the patency rate after vasovasostomy and the pregnancy rate is time dependent. In this study, we evaluated the influence of reproductive tract obstruction on expression of epididymal proteins and their restoration after patency. Adult male Wistar rats were studied 30,60 and 120 days after vasectomy, 30 days after vasovasostomy or after sham operations. Two-dimensional gel electrophoresis, mass-spectrometric technique, multidatabase search, Western blotting and real-time PCR were used to analyze the expression regulation of epididymal proteins. Total integrated intensity and total spot area of autoradiograms showed a consistent downward trend with time after obstruction, and this trend remained after patency. The intensity of the autoradiographic spots in three patency groups showed three trends: a downward trend, similar intensity and an upward trend compared with the correspondent obstruction group, respectively. Further verified experiments on human epididymis 2 (HE2), fertilization antigen-1 (FA-1), clusterin and PH20 demonstrated that compared with the correspondent obstruction group, the translation levels of HE2 and the mRNA transcription levels of HE2 showed an upward trend in patency groups, especially in the groups of obstruction for 60 days where the expression levels of HE2 were significantly upregulated after patency $(\boldsymbol{P}<0.05)$. Reproductive tract obstruction provokes a disregulation of gene expression in the epididymis and this disregulation remained after patency. Successful reversal may recover some proteins and the recovery is time dependent. Obstruction differentially alters mRNA transcription of different proteins and the content of proteins seemed to be easier to be influenced than the gene transcription. Asian Journal of Andrology (2013) 15, 105-109; doi:10.1038/aja.2012.64; published online 27 August 2012
\end{abstract}

Keywords: epididymal secretory proteins; vasectomy; vasovasostomy

\section{INTRODUCTION}

Vasectomy, a simple and reliable method of male contraception, has been widely accepted in the world. A growing number of men require vasectomy reversal because of the increasing divorce rate. Vasovasostomy by modern microsurgical techniques remains the standard technique for vasectomy reversal with which all other methods of vasectomy reversal are compared. If success is defined that sperm can be detected in ejaculate following reversal, patency is over $80 \%$ in most microsurgical series, with patency approaching $100 \%$ in some reports. ${ }^{1}$ However, pregnancies are obtained without assisted reproduction in $30 \%-75 \%$ of couples. ${ }^{2}$ In other words, about $25 \%$ of couples are affected. Male factors account for about $50 \%$ of couples with infertility. ${ }^{3}$ Approximately $10 \%-15 \%$ of infertile men suffer from azoospermia-complete absence of sperms in the ejaculate. Among these azoospermic patients, approximately $40 \%$ have complete obstruction in the ductal system and hence suffer from obstructive azoospermia. ${ }^{4}$ Vasoepididymostomy is performed for congenital, infectious, postvasectomy or idiopathic epididymal obstruction. Following this type of microsurgery, $20 \%-40 \%$ of couples achieve pregnancy through intercourse without the assistance of reproductive techniques. ${ }^{2}$ Similar to vasectomy reversal, the pregnancy rate is much lower than the patency rate. Studies have shown that vasectomy could have deleterious consequences on the epididymal function in cases of vasectomy reversal. It thus seems that vasectomy affects the expression of at least one gene, $P 34 \mathrm{H}$, involved in the maturation steps of sperm cells. Doiron et al. ${ }^{5}$ reported that the human epididymis 2 (HE2)-like mRNA predominantly expressed in the normal corpus epididymidis was significantly lowered 14 weeks after vasectomy. It is important to know whether epididymal protein synthesis and secretion are repaired by vasovasostomy and vasoepididymostomy. In this study, we describe our efforts to determine the effect of a more prolonged vasectomy on caput epididymal protein synthesis and secretion and whether spermatic duct reversal allows changes of protein synthesis and secretion to return to control conditions.

\section{MATERIALS AND METHODS}

Animal

Seventy adult male Wistar rats (200-250 g) were obtained from the Department of Laboratory Animal Science, Shanghai Medical College, Fudan University. The animal health was assessed on the day after the surgical procedure, and then regularly inspected until the epididymides were collected. All animal care and surgical procedures were performed in accordance with the guidelines of the animal care and use committee, Fudan University (Permit Number: 2007-0002). 


\section{Animal operation procedure}

Animals were anesthetized with $10 \%$ chloral hydrate $\left(300 \mathrm{mg} \mathrm{kg}^{-1}\right.$ of body weight) and subjected to vasectomy by transection of the vas deferens between two ligatures. Sham vasectomy was performed similarly, except that the suture was passed around the vas but not tied and that the vas was not transected. The animals with vasectomy were divided into three groups and studied 30,60 and 120 days after the operation, respectively. Three groups of rats received a vasovasostomy 30, 60 and 120 days after vasectomy, respectively, using four full thickness sutures and four superficial sutures. ${ }^{6}$ The animals were studied 30 days after vasovasostomy. Ten rats were used in every group.

\section{Confirmation of the vas deferens patency}

The patency was confirmed before the epididymis was removed by absence of spermatocele and vas deferens angiography from the anastomosis of proximal vas deferens. If the spermatocele was present or vas deferens angiography revealed the anastomosis was obstructed, the epididymis would be excluded from further experiments. According to the results, the epididymides were divided into six groups including obstruction for 30 days, obstruction for 60 days, obstruction for 120 days, patency after obstruction for 30 days, patency after obstruction for 60 days and patency after obstruction for 120 days. One group included six epididymal specimens. The epididymal protein quantification change was confirmed by comparing the proteins quantification in patency epididymides with the obstruction epididymides (obstruction $v s$. patency). The epididymides with different obstruction periods were also compared.

\section{Two-dimensional gel electrophoresis (2-DE)}

Samples of cell-free caput of the epididymis ( $50 \mathrm{mg}$ each) were used for 2-D electrophoresis (2-DE). The sham-operated group was used as the control. 2-DE was performed according to the methods described previously. ${ }^{7}$ We repeated each 2-DE experiment four times to confirm the spot patterns before proceeding with further analysis. Matching accuracy was $50 \%$. The amount of protein spots were defined as the percentage of the pixel intensity of the point in all pixels intensity of the gel. Isoelectric point and molecular weight were calculated by the one-dimensional calibration method.

\section{Protein identification by MALDI-TOF-MS}

The autoradiographic spots (proteins separated by 2-DE) which were visually noted on autoradiograms as being consistently secreted in sham-operated epididymides were selected for identification by MALDI-TOF-MS. The selected 2-DE spots were extracted and digested. The identification procedure was performed as described previously. ${ }^{8}$ The parameters of MALDI-TOF were set up as follows: positive ion-reflector mode; accelerating voltage was $20 \mathrm{kV}$; grid voltage was $64.5 \%$; mirror voltage ratio was $1.12 ; \mathrm{N}_{2}$-laser wavelength was $337 \mathrm{~nm}$; pulse width was $3 \mathrm{~ns}$; the number of laser shots was 50; acquisition mass range was 800-3000 Da; delay was $100 \mathrm{~ns}$; and vacuum degree was $4 \times 10^{7}$ torr. In the peptide mass fingerprinting map database searching, a Mascot Distiller was used to get the monoisotopic peak list from the raw mass spectrometry files. The monoisotopic peak list was used to search the Swiss-Prot or NCBInr databases with the Mascot search engine (http://www.matrixscience. com/). The searching parameter was set up as follows: the taxonomy was selected as all entries; enzyme was selected as trypsin; the mass tolerance was $\pm 50 \mathrm{ppm}$; the missed cleavage sites were allowed up to one; the fixed modifications were selected as carbamidomethyl (C); and the variable modification was selected as none.

\section{Western blotting}

Fifty micrograms of total protein was separated by 2-DE, as described above, and transferred to PVDF membranes (Millipore). Blots were incubated overnight at $4{ }^{\circ} \mathrm{C}$ with primary antibodies including rabbit anti-rat HE2 (1:1000; LifeSpan BioSciences, Seattle, WA, USA) rabbit anti-rat fertilization antigen-1 (FA-1, 1: 1000; Santa Cruz Biotechnology, Santa Cruz, CA, USA), mice anti-rat clusterin (1:1000; Santa Cruz) and rabbit anti-rat hyaluronidase PH-20 (1:1000, Santa Cruz). After three washes with PBS-Tween, blots were incubated for $2 \mathrm{~h}$ at $20{ }^{\circ} \mathrm{C}$ with secondary antibody labeled with HRP (Cell Signaling Technology, Beverly, MA, USA). The immune complexes were revealed by enhanced chemiluminescence and detected by X-ray films. Finally, the X-ray films were scanned and densitometric analysis was performed using the BioRad Quantity One software. Each reaction was performed in triplicate. Bands were corrected through gray scale scanning diameter using $\beta$ actin $(43 \mathrm{kD})$ as the internal reference. Six epididymal specimens were used for the experiment.

\section{Real-time fluorescence quantitative-PCR}

Fifty milligrams of recipient caput of the epididymis was sampled. Total RNA was extracted using a Trizol kit (Invitrogen, Shanghai, China) following the manufacturer's instructions. One microgram of the total RNA was subjected to reverse transcription of cDNA with transcriptase (MBI Fermentas) according to the manufacturer's instructions. Twenty nanograms of the resulting cDNA were then used in the real-time PCR step. The sequences of gene-specific primers designed and synthesized by Invitrogen Company are shown in Table 1. Each run was performed as described by the manufacturer's instructions. A negative control without cDNA template was run with every assay to assess the overall specificity. Each assay included duplicate reactions for each dilution and was repeated once. Standard curves were created using serial dilutions of genes. The gene expression values were normalized with the levels of the housekeeping gene $\beta$-actin. Melting curve analysis and analysis of the products by electrophoresis validated the PCR reactions. Six epididymal specimens were used for the experiment.

\section{Statistical analysis}

Data were shown as mean \pm s.d. Statistical analysis was conducted using SPSS 11.5 Statistical Software (IBM, New York, NY, USA) performing one-way ANOVA, with the significance level at $P<0.05$.

\section{RESULTS}

\section{Obstruction and patency rate}

Obstruction occurred in $100 \%$ of the vasectomized animals. The patency rates of the three vasovasostomy groups (vasovasostomy performed 30, 60 and 120 days after vasectomy) were $90 \%, 75 \%$ and $60 \%$, respectively.

\section{2-DE profiles of protein in the rat caput epididymidis}

The proteins of the epididymides were extracted for 2-DE analysis. Image analysis of 2-D autoradiograms demonstrated that the total integrated intensity and total spot area of autoradiograms showed a consistent downward trend after obstruction. Meanwhile, this trend remained after patency. The intensity of the autoradiographic spots in the patency groups showed a downward trend, similar intensity and an upward trend, respectively, compared with the corresponding obstruction groups (patency after obstruction for 30 days $v s$. obstruction for 30 days, patency after obstruction for 60 days $v s$. obstruction for 60 days, patency after obstruction for 120 days $v s$. obstruction for 120 days) (intensity data not shown). 
Table 1 The sequences of gene-specific primers for RT-PCR

\begin{tabular}{|c|c|c|c|c|}
\hline \multirow{2}{*}{$\frac{\text { Gene }}{\text { HE2 (SPAG11C) }}$} & \multicolumn{2}{|c|}{ The sequences of gene-specific primers } & \multirow{2}{*}{$\frac{\text { Temperature }\left({ }^{\circ} \mathrm{C}\right)}{58.9}$} & \multirow{2}{*}{$\frac{\text { Amplification length (bps) }}{118}$} \\
\hline & Forward: & 5'-CAGTGCCTCGTCCATTAACCACTT-3' & & \\
\hline & Reverse: & 5'-AGGGAAGCGTTTCACTCTGTGATG-3' & 58.9 & \\
\hline \multirow[t]{2}{*}{ FA-1 (NHP2-like protein 1) } & Forward: & 5'-TGGACCTTGTTCAGCAGTCGTGTA-3' & 59.9 & 111 \\
\hline & Reverse: & 5'-CGTCTGCTGCCATCACAATGAACT-3' & 59.5 & \\
\hline \multirow[t]{2}{*}{ Clusterin } & Forward: & 5'-ACCATCACAGACACCCACAGAAGT-3' & 59.9 & 110 \\
\hline & Reverse: & 5'-ACGGTGGTGTTTACTTCACCCGAT-3' & 60.2 & \\
\hline \multirow[t]{2}{*}{ Hyaluronidase $\mathrm{PH} 20$} & Forward: & 5'-ACCTGGATGAGAAACTGGACACCT-3' & 59.5 & 117 \\
\hline & Reverse: & 5'-TTGTGCTTTGGCTCTCACAGTAGC-3' & 59.3 & \\
\hline \multirow[t]{2}{*}{$\beta$-actin } & Forward: & 5'-AGCTGTGCTATGTTGCCCTAGACT-3' & 59.7 & 120 \\
\hline & Reverse: & 5'-ACCGCTCATTGCCGATAGTGATGA-3' & 60.1 & \\
\hline
\end{tabular}

Abbreviations: FA-1, fertilization antigen-1; HE2, human epididymis 2.

\section{Identification and bioinformatics analysis of differentially expressed proteins}

The extracted and digested 2-DE spots were identified as the proteins shown in Table 2. Nos. 1-8 and 10-11 autoradiographic spots were successfully identified, and no result was found in No. 9 autoradiographic spot. The intensity of all the autoradiographic spots demonstrated a downward trend after obstruction. Integrated with the intensity of the autoradiographic spots of 2-D, Nos. 1, 4, 7 and 8 autoradiographic spots, Nos. 2, 3, 5, 6 and 10, and No. 11 in the group after patency showed an upward trend, a downward trend and similar intensity compared with the correspondent group after obstruction, respectively (intensity data not shown).

\section{Western blotting}

The results of bands were demonstrated in Figure 1a. Compared with the sham-operated group, the expression levels of HE2, FA-1 and clusterin were significantly downregulated $(P<0.05$, one-way ANOVA) (Figure 1b-1d). The expression of $\mathrm{PH} 20$ showed similar levels after obstruction (Figure 1e). The expression levels of HE2 showed an upward trend after patency, especially in the group of obstruction for 60 days, the expression levels of HE2 were significantly upregulated after patency $(P<0.05$, one-way ANOVA) (Figure 1b). The expression levels of FA- 1 continued to decrease after patency in all the patency groups $(P<0.05$, one-way ANOVA) (Figure 1c). Clusterin and $\mathrm{PH} 20$ showed similar expression levels to the correspondent obstruction groups after patency in all the patency groups (Figure 1d and 1e).

\section{Real-time fluorescence quantitative-PCR}

The results were demonstrated in Figure 2. Compared with the shamoperated group, the mRNA transcription of FA-1, clusterin and PH20 showed similar levels in all the obstruction groups (Figure $\mathbf{2 b - 2 d}$ ). The mRNA transcription of FA-1, clusterin and PH20 demonstrated similar levels to the correspondent obstruction groups after patency in all patency groups (Figure $\mathbf{2 b - 2 d ) . ~ C o m p a r e d ~ w i t h ~ t h e ~ s h a m - o p e r a t e d ~}$ group, the mRNA transcription levels of HE2 demonstrated a downward trend after obstruction ( $P<0.05$, one-way ANOVA) (Figure 2a). The mRNA transcription levels of HE2 showed an upward trend after patency, especially in the group of obstruction for 60 days, and the mRNA transcription levels of HE2 were significantly upregulated after patency $(P<0.05$, one-way ANOVA) (Figure 2a).

\section{DISCUSSION}

Many studies have demonstrated that the proteins in the epididymis play important roles in sperm maturation. ${ }^{9,10}$ The dramatic recent progress made in the management of male infertility is largely attributable to the improved microsurgical techniques for the repair of reproductive tract obstruction. ${ }^{11}$ However, natural pregnancy occurs in only $15 \%-50 \%$ of these cases after repair of the reproductive tract obstruction. ${ }^{12,13}$ The pregnancy rate decreases with increasing time between obstruction of the reproductive tract and its surgical reversal, suggesting that sequelae to the male reproductive tract increases with time after surgical reversal. ${ }^{14-16}$ This study tried to demonstrate the expression change of epididymal proteins after obstruction and its surgical reversal.

Table 2 Identification of 11 selected differentially expressed proteins

\begin{tabular}{|c|c|c|c|c|c|c|c|}
\hline Spot No. & Protein name & UniProt ID & $\begin{array}{l}\text { Matching } \\
\text { peptides }\end{array}$ & $p l$ & $M W(D a)$ & $\begin{array}{l}\text { Sequence } \\
\text { coverage (\%) }\end{array}$ & Gene ID \\
\hline 1 & HE2 (SPAG11C) & Q1M189 & $4 / 11$ & 8.28 & 12556 & 40.90 & 652998 \\
\hline 2 & $\begin{array}{l}\text { Fertilin-beta (disintegrin and metalloproteinase } \\
\text { domain-containing protein 2, ADAM 2) }\end{array}$ & Q63202 & $13 / 16$ & 6.31 & 82311 & 53.61 & 56806 \\
\hline 3 & FA-1 (NHP2-like protein 1) & P55770 & $7 / 13$ & 4.31 & 14174 & 42.10 & 300092 \\
\hline 4 & Clusterin & P05371 & $10 / 19$ & 6.04 & 51375 & 51.50 & 24854 \\
\hline 5 & CRISP1 & P12020 & $5 / 16$ & 6.79 & 27847 & 35.60 & 64827 \\
\hline 8 & Chitinase-3-like protein 1 & Q9WTV1 & $6 / 15$ & 3.97 & 42392 & 32.50 & 89824 \\
\hline 9 & No result & & & & & & \\
\hline 10 & Fibronectin (Fn) & P04937 & $6 / 29$ & 9.61 & 272511 & 12.60 & 25661 \\
\hline 11 & G-protein coupled receptor 64 (HE6) & Q8CJ11 & $4 / 16$ & 5.13 & 110701 & 31.90 & 266735 \\
\hline
\end{tabular}

Abbreviations: CRISP1, cysteine-rich secretory protein 1; ERABP, epididymal retinoic acid-binding protein; FA-1, fertilization antigen-1; HE2, human epididymis 2; MW, molecular weight; pl, isoelectric point. 

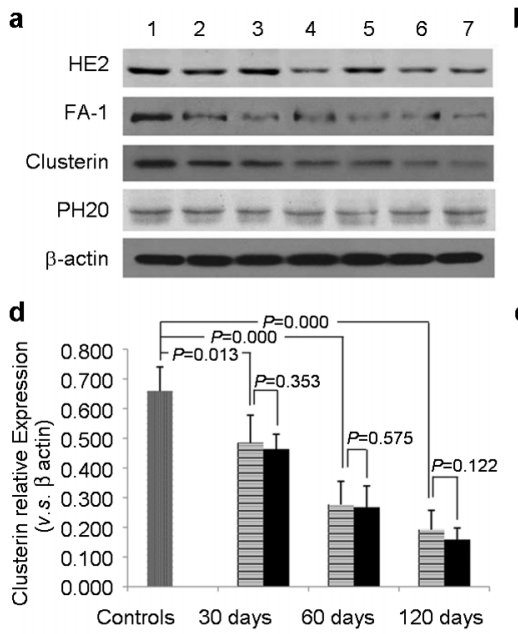

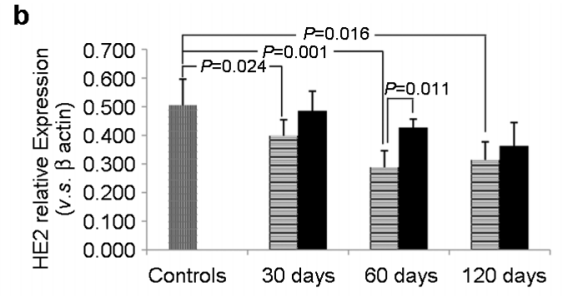

$\mathbf{e}$

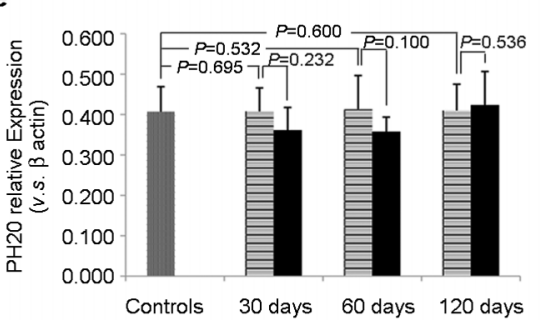

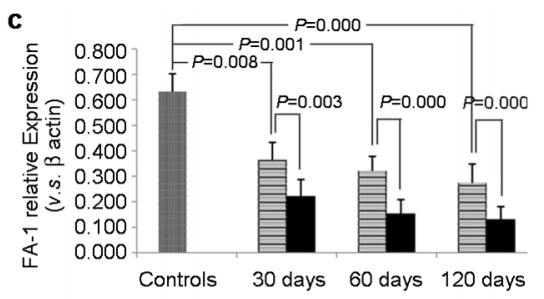

目 obstruction

- patency

Figure 1 The scanned X-ray films of Western blot (a) and the Western blot results of HE2 (b), FA-1 (c), clusterin (d) and PH2O (e). Lane 1: controls; lane 2: obstruction for 30 days; lane 3: patency 30 days after obstruction; lane 4: obstruction for 60 days; lane 5: patency 60 days after obstruction; lane 6: obstruction for 120 days; and lane 7: patency 120 days after obstruction. FA-1, fertilization antigen-1; HE2, human epididymis 2.

Image analysis of 2-D autoradiograms demonstrated that the total integrated intensity and total spot area of autoradiograms showed a consistent downward trend after reproductive tract obstruction, and this trend remained after patency. It is interesting that the total integrated intensity and total spot area of autoradiograms of patency after 60 days obstruction seem to be even lower than those of the group of obstruction for 120 days, which may be caused by the deposit of the proteins, since some studies have reported that morphological evaluation did not show significant remodeling of the caput epididymidal epithelium after reproductive tract obstruction. ${ }^{17}$ However, a consistent downward trend of epididymal proteins remained after vasovasostomy. We consider that the function of the epididymis continues to be altered or becomes worse after surgically successful vasovasostomy because of the injury caused by the two surgeries.

HE2

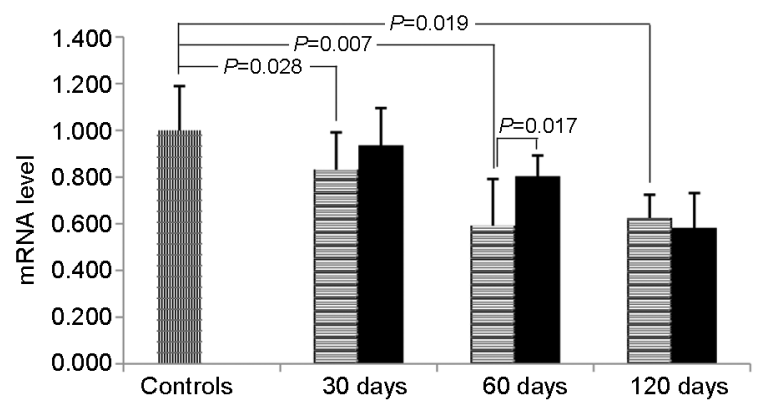

b

\begin{abstract}
FA-1
\end{abstract}

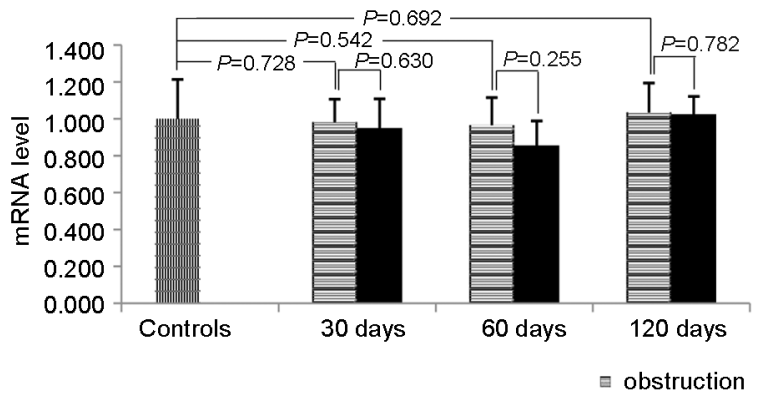

For the specific proteins, 10 epididymal proteins, including HE2, fertilin, sperm FA-1, clusterin, cysteine-rich secretory protein-1, epididymal retinoic acid-binding protein, $\mathrm{PH} 20$, chitinase 3-like protein 1, fibronectin and HE6, were identified by MALDI-TOF-MS. Among these proteins, chitinase 3 -like protein 1 which is associated with a variety of immune and inflammatory diseases, tissue remodeling and fibrosis, showed an upward trend after patency. ${ }^{18,19}$ Therefore, immune and inflammatory diseases, tissue remodeling and fibrosis might be aggravated by surgical vasovasostomy and the luminal microenvironment might be consequently altered.

The disadvantages of 2-D may include less reproducibility, difficulty to separate low abundance proteins, acidic and basic proteins, very large and very small proteins and hydrophobic proteins. ${ }^{20}$ Therefore, the result of 2-D autoradiograms needed to be confirmed by quantitative
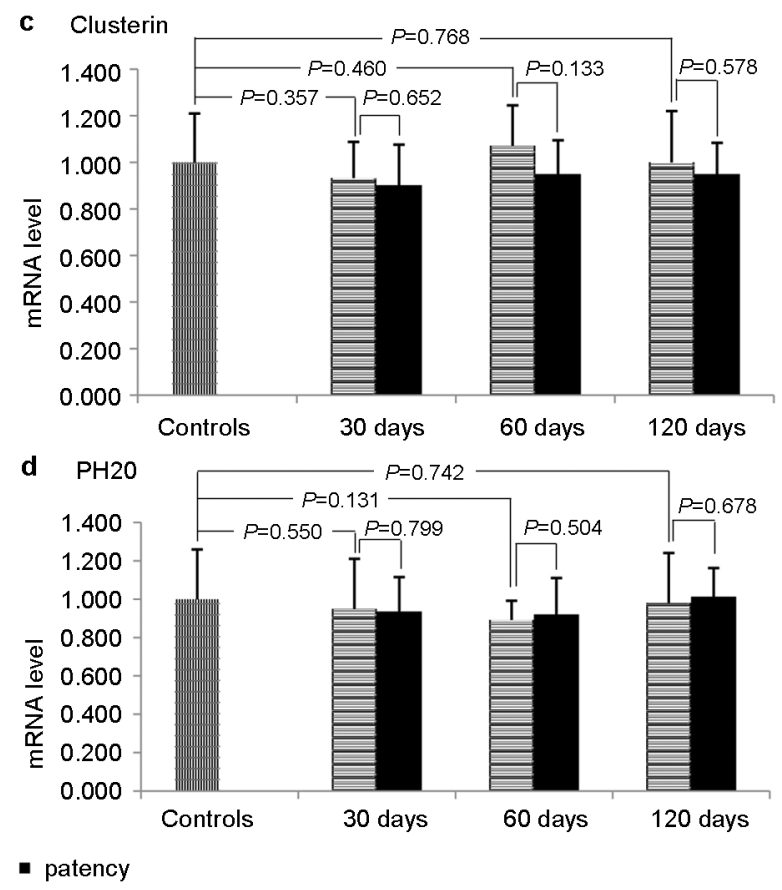

Figure 2 The RT-PCR results of HE2 (a), FA-1 (b), clusterin (c) and PH2O (d). FA-1, fertilization antigen-1; HE2, human epididymis 2. 
experimental procedures. Western blot and RT-PCR were used to quantitatively analyze the expression of specific proteins. The protein expression levels of HE2, FA-1, clusterin and PH20 were selected for verification by Western blot and RT-PCR because they play important roles in sperm maturation and fertilization process. ${ }^{21-29}$

Western blot demonstrated that the content of HE2 was downregulated after reproductive tract obstruction and the downward trend was more obvious with increasing time of obstruction. Another study by Légaré et al. ${ }^{21}$ showed that vasectomy did not influence expression of HE2 in the human epididymis. Because we also found that the content of HE2 in the group of obstruction for 30 days was similar to the sham-operated group, it is possible that the interval of obstruction was not long enough in Légaré et al.'s study. Patency may recover the expression level of HE2, especially in the group of obstruction for 60 days. However, the expression level of HE2 in the group of obstruction for 120 days cannot be recovered by reproductive tract patency. RTPCR also demonstrated a similar result. The recovery of HE2 could be time dependent. This could have important clinical significance in cases of vasectomy reversal. Western blot demonstrated the content of FA-1, clusterin and PH20 had different situations. In other words, the impact of obstruction and patency to the specific epididymal proteins was different. The reason might be the different expression and metabolism process of individual proteins. However, the RNA levels of FA-1, clusterin and PH20 were similar among all of the groups. The reason might be that although the reproductive tract obstruction could affect the expression of the epididymal gene necessary for the acquisition of sperm fertilizing ability, the content of proteins seemed to be easier to be influenced than the gene transcription.

Limitations of this study include the fact that the expression changes of epididymal proteins did not integrate with the fertile rate of rats to analyze the impact on pregnancy rate after patency. Moreover, although the irreversible modifications in gene expression induced by reproductive tract obstruction may explain the discrepancy between a surgically successful vasovasostomy and the pregnancy rate, clinical observations suggest that it may not be as important for sperm maturation, as is the case for laboratory animals. ${ }^{30}$ Therefore, the study of proteome and transcriptome should be performed in the epididymis of humans rather than that in the epididymis of laboratory animals.

\section{CONCLUSIONS}

The overall results suggest that reproductive tract obstruction provokes a disregulation of gene expression in the epididymis and this disregulation remained after patency. For some individual proteins, the effect of reproductive tract obstruction could be time dependent. Successful reversal may recover some proteins and the shorter the interval of reproductive tract obstruction is, the better the epididymal proteins recovers. Obstruction differentially alters mRNA expression of different proteins and the content of proteins seemed to be easier to be influenced than the gene transcription.

\section{AUTHOR CONTRIBUTIONS}

$\mathrm{XW}$ and BKL conceived and designed the experiments. BKL performed the experiments. BKL, CXL, SBZ and HLL analyzed the data. BKL, XW, CXL, SBZ, HLL, LPL and ABX wrote the paper.

\section{COMPETING FINANCIAL INTERESTS}

The authors would declare no competing financial interests.

\section{ACKNOWLEDGMENTS}

The authors would like to acknowledge the National Natural Science Foundation of China (No. 30872595) and National Basic Research Program
(973) of China (No. 2011CB944503) for their financial support. We also thank Dr Yong Lu for his assistance with skillful microsurgery.

1 Bolduc S, Fischer MA, Deceuninck G, Thabet M. Factors predicting overall success: a review of 747 microsurgical vasovasostomies. Can Urol Assoc J 2007; 1: 388-94.

2 Practice Committee of the American Society for Reproductive Medicine. Report on management of obstructive azoospermia. Fertil Steril 2006; 86: S259-63.

3 Lee R, Li PS, Schlegel PN, Goldstein M. Reassessing reconstruction in the management of obstructive azoospermia: reconstruction or sperm acquisition? Urol Clin North Am 2008; 35: 289-301.

4 Practice Committee of American Society for Reproductive Medicine in collaboration with Society for Male Reproduction and Urology. The management of infertility due to obstructive azoospermia. Fertil Steril 2008; 90: 121S-4S

5 Doiron K, Légaré C, Saez F, Sullivan R. Effect of vasectomy on gene expression in the epididymis of cynomolgus monkey. Biol Reprod 2003; 68: 781-8.

6 Turner TT, Riley TA, Vagnetti M, Flickinger CJ, Caldwell JA et al. Postvasectomy alterations in protein synthesis and secretion in the rat caput epididymidis are not repaired after vasovasostomy. J Androl 2000; 21: 276-90.

7 Li C, Tan YX, Zhou H, Ding SJ, Li SJ et al. Proteomic analysis of hepatitis B virusassociated hepatocellular carcinoma: Identification of potential tumor markers. Proteomics 2005; 5: 1125-39.

8 Ding SJ, Li Y, Tan YX, Jiang MR, Tian B et al. From proteomic analysis to clinical significance: overexpression of cytokeratin 19 correlates with hepatocellular carcinoma metastasis. Mol Cell Proteomics 2004; 3: 73-81.

9 Dacheux JL, Gatti JL, Dacheux F. Contribution of epididymal secretory proteins for spermatozoa maturation. Microsc Res Tech 2003; 61: 7-17.

10 Yuan $\mathrm{H}$, Liu A, Zhang L, Zhou H, Wang $\mathrm{Y}$ et al. Proteomic profiling of regionalized proteins in rat epididymis indicates consistency between specialized distribution and protein functions. J Proteome Res 2006; 5: 299-307.

11 Goldstein M, Tanrikut C. Microsurgical management of male infertility. Nat Clin Pract Urol 2006; 3: 381-91.

12 Thimon V, Calvo E, Koukoui O, Légaré C, Sullivan R. Effects of vasectomy on gene expression profiling along the human epididymis. Biol Reprod 2008; 79: 262-73.

13 Feber KM, Ruiz HE. Vasovasostomy: macroscopic approach and retrospective review. Tech Urol 1999; 5: 8-11.

14 Belker AM, Thomas AJ Jr, Fuchs EF, Konnak JW, Sharlip ID. Results of 1,469 microsurgical vasectomy reversals by the Vasovasostomy Study Group. J Urol 1991 145: 505-11.

15 Abdelmassih V, Balmaceda JP, Tesarik J, Abdelmassih R, Nagy ZP. Relationship between time period after vasectomy and the reproductive capacity of sperm obtained by epididymal aspiration. Hum Reprod 2002; 17: 736-40.

16 Lopushnyan NA, Walsh TJ. Surgical techniques for the management of male infertility. Asian J Androl 2012; 14: 94-102.

17 Flickinger CJ, Herr JC, Sisak JR, Howards SS. Ultrastructure of epididymis interstitial reactions following vasectomy and vasovasostomy. Anat Rec 1993; 235: 61-73.

18 Choi J, Lee HW, Suk K. Plasma level of chitinase 3-like 1 protein increases in patients with early Alzheimer's disease. J Neurol 2011; 258: 2181-5.

19 Lee CG, Da Silva CA, Dela Cruz CS, Ahangari F, Ma B et al. Role of chitin and chitinase/ chitinase-like proteins in inflammation, tissue remodeling, and injury. Annu Rev Physiol 2011; 73: 479-501.

20 Arthur JM. Proteomics. Curr Opin Nephrol Hypertens 2003; 12: 423-30.

21 Légaré $C$, Verville N, Sullivan R. Vasectomy influences expression of HE1 but not HE2 and HE5 genes in human epididymis. J Androl 2004; 25: 30-43.

22 Yenugu S, Hamil KG, Birse CE, Ruben SM, French FS et al. Antibacterial properties of the sperm-binding proteins and peptides of human epididymis 2 (HE2) family, salt sensitivity, structural dependence and their interaction with outer and cytoplasmic membranes of Escherichia coli. Biochem J 2003; 372: 473-83.

23 Yenugu S, Hamil KG, French FS, Hall SH. Antimicrobial actions of the human epididymis 2 (HE2) protein isoforms, HE2alpha, HE2beta1 and HE2beta2. Reprod Biol Endocrinol 2004; 2: 61.

$24 \mathrm{Na} \mathrm{RH}, \mathrm{Xu}$ RG. The expression of sperm fertilization antigen-1 (FA-1) mRNA in the testis and epididymis of ram. Anim Hus Vet Med 2006; 38: 1-3.

25 Lavers AE, Swanlund DJ, Hunter BA, Tran ML, Pryor JL et al. Acute effect of vasectomy on the function of the rat epididymal epithelium and vas deferens. J Androl 2006; 27: 826-36.

26 Griffiths GS, Galileo DS, Aravindan RG, Martin-DeLeon PA. Clusterin facilitates exchange of glycosyl phosphatidylinositol-linked SPAM1 between reproductive luminal fluids and mouse and human sperm membranes. Biol Reprod 2009; 81: 562-70.

27 Martin-Deleon PA. Germ-cell hyaluronidases: their roles in sperm function. Int $J$ Androl 2011; 34: e306-18.

28 Zhang $\mathrm{H}$, Jones R, Martin-DeLeon PA. Expression and secretion of rat SPAM1 (2B1 or PH 20 ) in the epididymis: role of testicular lumicrine factors. Matrix Biol 2004; 22: 653-61.

29 Morin G, Sullivan R, Laflamme I, Robert C, Leclerc P. SPAM1 isoforms from two tissue origins are differentially localized within ejaculated bull sperm membranes and have different roles during fertilization. Biol Reprod 2010; 82: 271-81.

30 Sullivan R, Legare C, Thabet M, Thimon V. Gene expression in the epididymis of normal and vasectomized men: what can we learn about human sperm maturation? $J$ Androl 2011; 32: 686-97. 